\title{
Outcomes from salvage chemotherapy or pembrolizumab beyond progression with or without local ablative therapies for advanced non-small cell lung cancers with PD-L1 $\geq 50 \%$ who progress on first-line immunotherapy: real-world data from a European cohort
}

\author{
Giulio Metro ${ }^{1}$, Alfredo Addeo ${ }^{2}$, Diego Signorelli ${ }^{3}$, Alessio Gili ${ }^{4}$, Panagiota Economopoulou ${ }^{5}$, Fausto Roila ${ }^{1}$, \\ Giuseppe Banna ${ }^{6}$, Alessandro De Toma ${ }^{3}$, Juliana Rey $\mathrm{Cobo}^{2}$, Andrea Camerini ${ }^{7}$, Athina Christopoulou ${ }^{8}$, \\ Giuseppe Lo Russo ${ }^{3}$, Marco Banini ${ }^{1}$, Domenico Galetta ${ }^{9}$, Beatriz Jimenez ${ }^{10}$, Ana Collazo-Lorduy ${ }^{10}$, \\ Antonio Calles ${ }^{11}$, Panagiotis Baxevanos ${ }^{12}$, Helena Linardou ${ }^{13}$, Paris Kosmidis ${ }^{14}$, Marina C. Garassino ${ }^{3}$, \\ Giannis Mountzios ${ }^{15}$
}

\begin{abstract}
${ }^{1}$ Medical Oncology, Santa Maria della Misericordia Hospital, Azienda Ospedaliera di Perugia, Perugia, Italy; ${ }^{2}$ Department of Oncology, Geneva University Hospital, Geneva, Switzerland; ${ }^{3}$ Medical Oncology Department, Fondazione IRCCS, Istituto Nazionale Tumori di Milano, Milano, Italy; ${ }^{4}$ Public Health Section, Department of Experimental Medicine, University of Perugia, Perugia, Italy; ${ }^{5}$ Oncology Department, Attikon University Hospital, Athens, Greece; ${ }^{6}$ Medical Oncology, Ospedale Cannizzaro, Catania, Italy; ${ }^{7}$ U.O.C. Oncologia, Ospedale Versilia, Lido di Camaiore (LU), Italy; ${ }^{8}$ Medical Oncology, Agios Andreas General Hospital of Patras, Patras, Greece; ${ }^{9}$ Medical Thoracic Oncology Unit, IRCCS Istituto Tumori “Giovanni Paolo II”, Bari, Italy; ${ }^{10}$ Medical Oncology, Hospital Universitario HM Sanchinarro, Madrid, Spain; ${ }^{11}$ Division of Medical Oncology, Hospital General Universitario Gregorio Marañón, Madrid, Spain; ${ }^{12}$ Second Department of Medical Oncology, Saint Savvas Anti-Cancer Hospital, Athens, Greece; ${ }^{13}$ First Department of Medical Oncology, Metropolitan Hospital, Athens, Greece; ${ }^{14}$ Second Department of Medical Oncology, Hygeia Hospital, Athens, Greece; ${ }^{15}$ Second Department of Medical Oncology, Henry Dunant Hospital Center, Athens, Greece

Contributions: (I) Conception and design: G Metro; (II) Administrative support: G Metro, G Mountzios; (III) Provision of study materials or patients: G Metro, A Addeo, D Signorelli, P Economopoulou, F Roila, G Banna, A De Toma, J Rey Cobo, A Camerini, A Christopoulou, G Lo Russo, M Banini, D Galetta, B Jimenez, A Collazo-Lorduy, A Calles, P Baxevanos, H Linardou, P Kosmidis, MC Garassino, G Mountzios; (IV) Collection and assembly of data: G Metro, A Gili, G Mountzios; (V) Data analysis and interpretation: G Metro, A Addeo, D Signorelli, A Gili, G Banna, G Mountzios; (VI) Manuscript writing: All authors; (VII) Final approval of manuscript: All authors.

Correspondence to: Giulio Metro, MD. Medical Oncology, Santa Maria della Misericordia Hospital, Azienda Ospedaliera di Perugia, via Dottori, 1, 06156, Perugia, Italy. Email: giulio.metro@yahoo.com.
\end{abstract}

Background: In this real-world multicenter study we addressed the activity of post-progression anticancer treatments after first-line pembrolizumab in advanced non-small cell lung cancer (NSCLC) patients with $\mathrm{PD}-\mathrm{L} 1 \geq 50 \%$.

Methods: Clinico-pathological data of PD-L $1 \geq 50 \%$ advanced NSCLCs who failed first-line pembrolizumab were collected in 14 Oncologic Centers from different European countries. Types of subsequent anticancer treatment and outcomes on salvage chemotherapy or pembrolizumab beyond progression with or without the addition of local ablative therapies were reported.

Results: Out of 173 patients, 100 had progressed on pembrolizumab, of which 60 patients $(60 \%)$ met eligibility criteria and were treated with either salvage chemotherapy (42/60, 70\%) or pembrolizumab beyond progression $(18 / 60,30 \%)$. Overall, median age was 66 years, $63.3 \%$ were male, $60.0 \%$ had a performance status of $0-1,88.3 \%$ were smokers and $61.7 \%$ had adenocarcinoma histology. In patients evaluable for response, objective response rate to salvage chemotherapy was $41.9 \%$, with no significant difference according to the type of regimen (42.9\% for platinum-based and $40.0 \%$ for single-agent chemotherapy). Median progression-free survival (PFS) to salvage chemotherapy was 4.5 months. Among patients treated with pembrolizumab beyond progression, 13 out of 18 patients $(72.2 \%)$ had progressive disease in $\leq 2$ organ sites, of whom $9(69.2 \%)$ were managed with the addition of local ablative therapies consisting of radiation at progressive lesion(s). No significant difference was noted in terms of post-progression survival between the 
salvage chemotherapy and the pembrolizumab beyond progression groups of patients (6.9 versus 8.1 months, respectively, $\mathrm{P}=0.08)$.

Conclusions: In PD-L1 $\geq 50 \%$ advanced NSCLCs who progress on first-line pembrolizumab, salvage chemotherapy is associated with a remarkable anticancer activity, while select patients may benefit from continuation of pembrolizumab beyond progression, with the possible addition of local ablative radiotherapy in oligoprogressive cases.

Keywords: Local ablative therapy; non-small cell lung cancer (NSCLC); PD-L1 $\geq 50 \%$; pembrolizumab beyond progression; salvage chemotherapy

Submitted Aug 04, 2019. Accepted for publication Nov 26, 2019.

doi: $10.21037 /$ jtd.2019.12.23

View this article at: http://dx.doi.org/10.21037/jtd.2019.12.23

\section{Introduction}

Ever since its introduction for clinical use, immunotherapy with anti-programmed cell death 1 (PD-1) or antiprogrammed cell death ligand 1 (PD-L1) has revolutionized the treatment of patients with advanced non-small cell lung cancer (NSCLC) with no actionable mutations in the epidermal growth factor receptor (EGFR) or anaplastic lymphoma kinase (ALK) genes (1). In fact, while early trials demonstrated the superiority of anti-PD-1/PD-L1 agents over second-line chemotherapy in platinum-pretreated patients (2-5), more recent studies have shown that inhibition of PD-1 axis is also effective as first-line treatment (6-8).

PD-L1 expression levels on tumor cells have emerged as a biologic predictor of response to PD-1 axis inhibitor therapy, with a recent phase 3 trial showing improved survival with the anti-PD-1 agent pembrolizumab over platinum-based chemotherapy for treatment-naïve patients with a PD-L1 expression $\geq 50 \%(6,7)$. These results led to a paradigm change in the treatment algorithm of advanced NSCLCs, which led to the use of pembrolizumab instead of chemotherapy as first-line treatment of patients with PDL1 expression $\geq 50 \%$. In this context, interest has partly shifted to subsequent anticancer treatment to be offered after progression on pembrolizumab. In fact, it has not yet been fully elucidated to what extent PD-L1 $\geq 50 \%$ patients would benefit from salvage chemotherapy administered after pembrolizumab.

Characterizing the activity of salvage chemotherapy administered sequentially after pembrolizumab is of particular relevance in view of the great efficacy recently shown by pembrolizumab in combination with chemotherapy as first-line treatment irrespective of PD-L1 expression levels (8). For the same reason, as many patients will be exposed to the combination of pembrolizumab with chemotherapy in the first-line setting, treatment with pembrolizumab beyond progression has become an area of interest. However, data on the potential benefits of PD-1 axis inhibitors given beyond progression in NSCLC have been limited so far and mostly confined to the setting of patients pretreated with platinum-based chemotherapy (9-11). Therefore, at the present time, data on the use of pembrolizumab beyond progression in patients with PD-L1 $\geq 50 \%$ are scant, and the clinical benefit of such an approach is unknown. In addition, although local ablative therapies represent a consolidated approach in oligoprogressive patients with oncogene-addicted NSCLC treated with a tyrosine kinase inhibitor (12), the impact of local treatments in patients who continue treatment with $\mathrm{PD}-1$ axis inhibitors beyond progression has been poorly addressed.

On this basis, we ran a real-world multicenter study in advanced NSCLC patients with PD-L1 $\geq 50 \%$ in which we described the types of post-progression anticancer treatment and the clinical outcome after first-line pembrolizumab, focusing on strategies such as salvage chemotherapy and pembrolizumab beyond progression. In addition, in the latter group of patients, we focused on those individuals who received local ablative therapies in order to address the relevance of such a strategy in the pembrolizumab beyond progression group.

\section{Methods}

\section{Study population}

This retrospective study aimed at describing the types and 


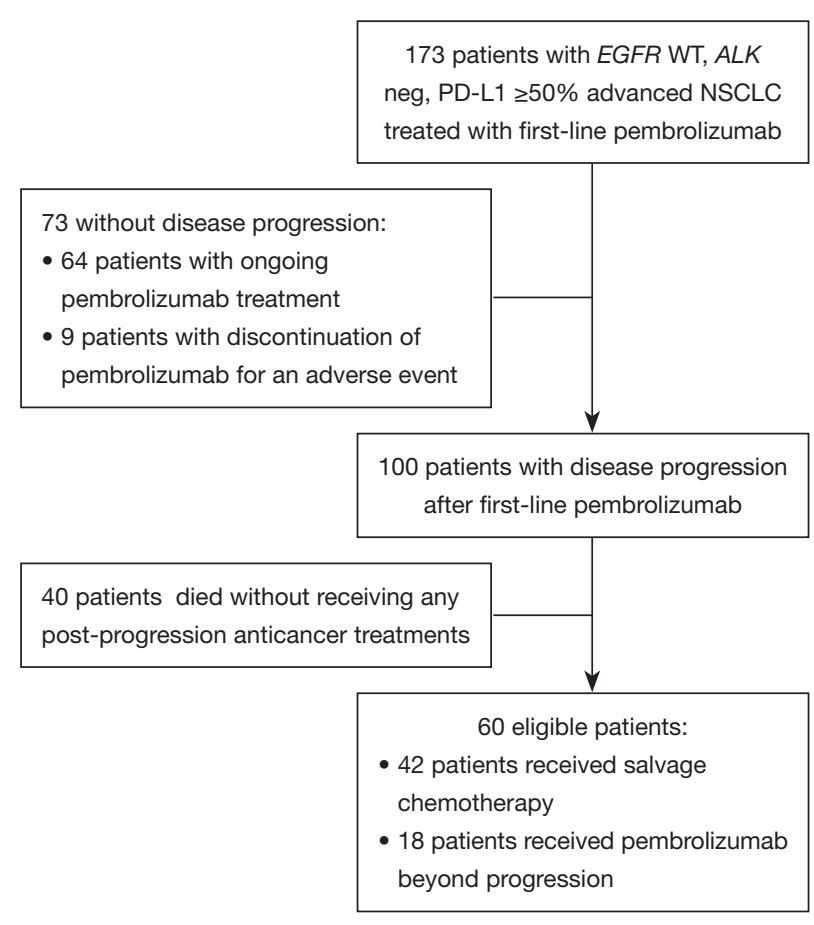

Figure 1 Study flowchart. WT, wild type; NSCLC, non-small cell lung cancer.

outcomes of subsequent anticancer treatments received after progression on first-line pembrolizumab in non-oncogene addicted advanced NSCLC patients with PD-L1 $\geq 50 \%$. The medical charts were reviewed and data were extracted from 14 Oncologic Centers operating in four different countries (Greece $\mathrm{n}=7$; Italy $\mathrm{n}=5$; Switzerland $\mathrm{n}=1$; Spain $\mathrm{n}=1$ ). As this was a real-life study, there were no clinical or pathological restrictions for patient enrollment, provided that eligible patients had been treated with first-line pembrolizumab, and had an EGFR wild type, $A L K$ negative, and PD-L $1 \geq 50 \%$ biological profile of the tumor (as per local assessment). Disease progression on pembrolizumab was defined as progressive disease (PD) as per RECIST 1.1 that occurred during treatment or within 6 months of the last dose of pembrolizumab.

The following data were collected: clinico-pathological characteristics, types of post-progression anticancer treatment, response to first-line pembrolizumab and to salvage chemotherapy as per RECIST 1.1 [complete response (CR), partial response (PR), stable disease (SD) and $P D$, with overall response rate (ORR) being the sum of $C R$ and $P R$, and disease control rate being the sum of $\mathrm{CR}, \mathrm{PR}$ and $\mathrm{SD}$ ], progression-free survival (PFS) of salvage chemotherapy (calculated from the time of initiation of treatment to the radiographic evidence of disease progression or death of the patient in the absence of disease progression, with alive patients without documented radiographic progression being censored at the time of last follow-up). In addition, post-progression survival (PPS) was evaluated for either the salvage chemotherapy and the pembrolizumab beyond progression groups of patients (calculated from the date of progression on pembrolizumab to death of the patient from any cause, with alive patients being censored at the time of the last follow-up). The collection of data on local ablative therapies was limited to patients who were treated with pembrolizumab beyond progression as this was among the outcomes of the present analysis. Since this was a real-world study, the decision on whether patients progressing on first-line pembrolizumab were treated with salvage chemotherapy or pembrolizumab beyond progression was at physicians' discretion.

\section{Statistical analysis}

Descriptive statistics were performed using frequencies, percentages, frequency tables for categorical variables, median and range for quantitative variables. Non-parametric Mann-Whitney test was performed to compare continuous variable with no normal distribution. Categorical variables were evaluated by chi-square analysis or Fisher's exact test where appropriate.

PFS and PPS were analyzed according to KaplanMeier method and survival curves were compared using the log-rank test. Cox model was used to estimate hazard ratio and related $95 \%$ confidence interval (CI). Given the retrospective nature of the study, statistical significance should be used in an exploratory view and median time estimation with their 95\% CI: reported to better interpret the data. All estimates were achieved using STATA 14.2 (Stata Corp Ltd., College Station, TX, USA).

\section{Results}

\section{Patients}

Figure 1 shows study flow-chart. Out of 173 patients treated with first-line pembrolizumab from January 2017 to March 2019, 100 patients experienced PD. Of them, 40 patients did not receive any subsequent anticancer treatments because of rapid deterioration of clinical conditions, while 60 patients received a post-progression treatment. Table 1 lists the types 
Table 1 Post-progression anticancer treatments

\begin{tabular}{|c|c|}
\hline $\begin{array}{l}\text { Types of post-progression } \\
\text { anticancer treatment }\end{array}$ & Total patients $(n=60)(\%)$ \\
\hline Salvage chemotherapy & $42(70.0)$ \\
\hline Platinum-based chemotherapy* & 31 out of $42(73.8)$ \\
\hline Mono-chemotherapy** & 11 out of $42(26.2)$ \\
\hline Pembrolizumab beyond progression & $18(30.0)$ \\
\hline \multicolumn{2}{|c|}{$\begin{array}{l}\text { *, } n=12 \text { carboplatin/pemetrexed; } n=5 \text { carboplatin/gemcitabine; } \\
\mathrm{n}=4 \text { cisplatin/pemetrexed; } \mathrm{n}=3 \text { carboplatin/paclitaxel; } \mathrm{n}=3 \\
\text { carboplatin/nab-paclitaxel; } \mathrm{n}=2 \text { carboplatin/paclitaxel/ } \\
\text { bevacizumab; } \mathrm{n}=1 \text { cisplatin/gemcitabine; } \mathrm{n}=1 \text { carboplatin. }{ }^{* *} \text {, } \\
\mathrm{n}=7 \text { gemcitabine; } \mathrm{N}=4 \text { docetaxel. }\end{array}$} \\
\hline
\end{tabular}

of subsequent anticancer treatments, consisting of salvage chemotherapy in 42 patients and pembrolizumab beyond progression in 18 cases. Platinum-based chemotherapy was delivered in approximately three quarters of patients who received salvage chemotherapy, with platinum/pemetrexed being the most commonly administered chemotherapeutic regimen. Table 2 shows the clinical characteristics of the 60 patients according to whether they received salvage chemotherapy or pembrolizumab beyond progression. No significant differences were seen between the two groups, except: (I) higher median age reported in the pembrolizumab beyond progression group (71 years versus 64 years, borderline significance); (II) more patients in the salvage chemotherapy group had adenocarcinoma histology (69.0\% versus $44.5 \%$, borderline significance); (III) more patients in the pembrolizumab beyond progression group experienced a PR or SD as best response to prior pembrolizumab ( $77.8 \%$ versus $47.6 \%$ ); (IV) the same group also had longer median duration of prior pembrolizumab (5.9 versus 3.6 months).

At the time of this analysis (June 2019), median followup time calculated from the date of progression to first-line pembrolizumab (based on the objectives of this analysis) was 5.7 months (range, $0.7-16.0$ months) in the salvage chemotherapy group and 5.5 months (range, 1.2-20.9 months) in the pembrolizumab beyond progression group.

\section{Efficacy of salvage chemotherapy}

Among the 42 patients who received salvage chemotherapy, 31 had at least one follow-up radiographic assessment, and were therefore evaluable for activity. Table 3 lists the activity of salvage chemotherapy. Overall, an ORR was observed in $41.9 \%$ of patients, with a disease control rate of $54.8 \%$. Thirty-three patients had died or had disease progression, and the median PFS was 4.5 months (95\% CI: 3.1-6.5 months) (Figure 2A).

No significant difference was seen in terms of ORR between the 23 patients who were treated with platinumbased chemotherapy versus the ten patients who received single-agent chemotherapy, the ORR being $42.9 \%$ and $40.0 \%$, respectively (Table 3).

We performed an exploratory analysis to determine whether any clinical or pathologic features were associated with PFS. However, at the univariate analysis none of the variables considered (age; male versus female; PS 0-1 versus 2; never smokers versus ever smokers; adenocarcinoma versus other histologies; brain metastases versus no brain metastases; PD-L1 $\geq 50-74 \%$ versus $\geq 75-100 \%$; PR or $\mathrm{SD}$ versus $\mathrm{PD}$ on prior pembrolizumab) were significantly associated with clinical outcome; these evidences were confirmed in the multivariate approach.

\section{Pembrolizumab beyond progression}

Eighteen patients were treated with pembrolizumab beyond progression. Table 4 lists the patients for whom it was deemed clinically appropriate by the investigator to continue on pembrolizumab beyond progression with or without the addition of local ablative therapies. Overall, local treatment was delivered in 11 patients, and consisted of pericardial drainage in two patients who had developed symptomatic pericardial effusions and local ablative radiotherapy in the remainder 9 cases.

Among the 13 patients with PD in $\leq 2$ organ sites, local ablative radiotherapy at progressive organ sites/lesion(s) was administered in 9 cases (69.2\%). It consisted of whole brain radiotherapy in four cases of isolated central nervous system progression with multiple $(>3)$ brain metastasis $(\mathrm{n}=3$ patients with new brain metastases, $n=1$ patient with pre-existing brain metastases) and stereotactic radiation to extra-cranial progressive lesion(s) in 5 other cases $(\mathrm{n}=4$ patients at a single progressive lesion, $\mathrm{n}=1$ patient at two progressive lesions).

\section{Post-progression survival}

Thirty-two patients in the salvage chemotherapy group and six patients in the pembrolizumab beyond progression group had died, respectively. Overall, median PPS was 8.2 months (95\% CI: 4.8-9.1 months): median PPS was 6.9 months (95\% CI: 4.0-8.7 months) in the salvage chemotherapy and 8.1 months (95\% CI: $5.8-\mathrm{NR}$ ) in the 
Table 2 Characteristics of patients

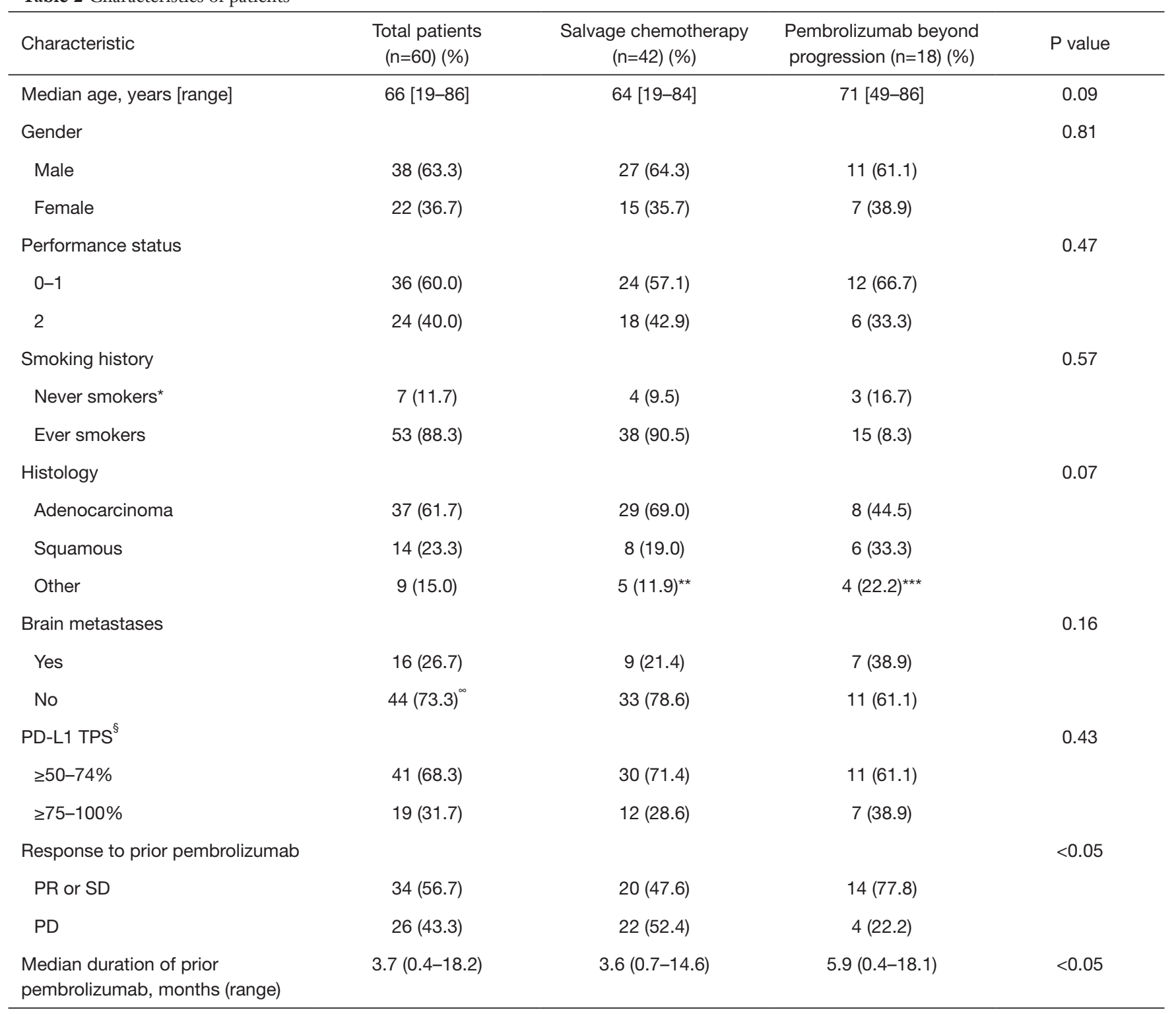

*, less than 100 cigarettes in the lifetime; ${ }^{*}, 3$ non-small cell lung cancers not otherwise specified, 1 large cell carcinoma, 1 adenosquamous; ${ }^{\star \star \star}, 2$ non-small cell lung cancers not otherwise specified, 2 sarcomatoid carcinomas; ${ }^{\infty}$, includes 6 patients not staged for brain metastases, 3 in each group; ${ }^{\S}$, as assessed by immunohistochemistry (Dako 22C3 PharmDx). PD, progressive disease; PR, partial response; SD, stable disease; TPS, tumor proportion score.

pembrolizumab beyond progression groups of patients, $\mathrm{P}=0.08$ (Figure $2 \mathrm{~B}$ ). Among the nine patients with $\mathrm{PD}$ in $\leq 2$ organ sites who were treated with pembrolizumab beyond progression with the addition of local ablative radiotherapy the PPS rates at 6 and 12 months were $88.9 \%$ (95\% CI: 43.3-98.4\%) and 71.1\% (95\% CI: 23.3-92.3\%), respectively, while the same values for the remainder nine patients were 63.5\% (95\% CI: 23.8-86.6\%) and 0\%, respectively (Table 4).

\section{Discussion}

In this analysis, we focused on the types of postprogression anticancer treatments and on the clinical outcome of subsequent anticancer therapies after firstline pembrolizumab in PD-L1 $\geq 50 \%$ advanced NSCLC patients. This clinical question is highly relevant in view of the existence of two competing first-line standards of 
Table 3 Activity of salvage chemotherapy

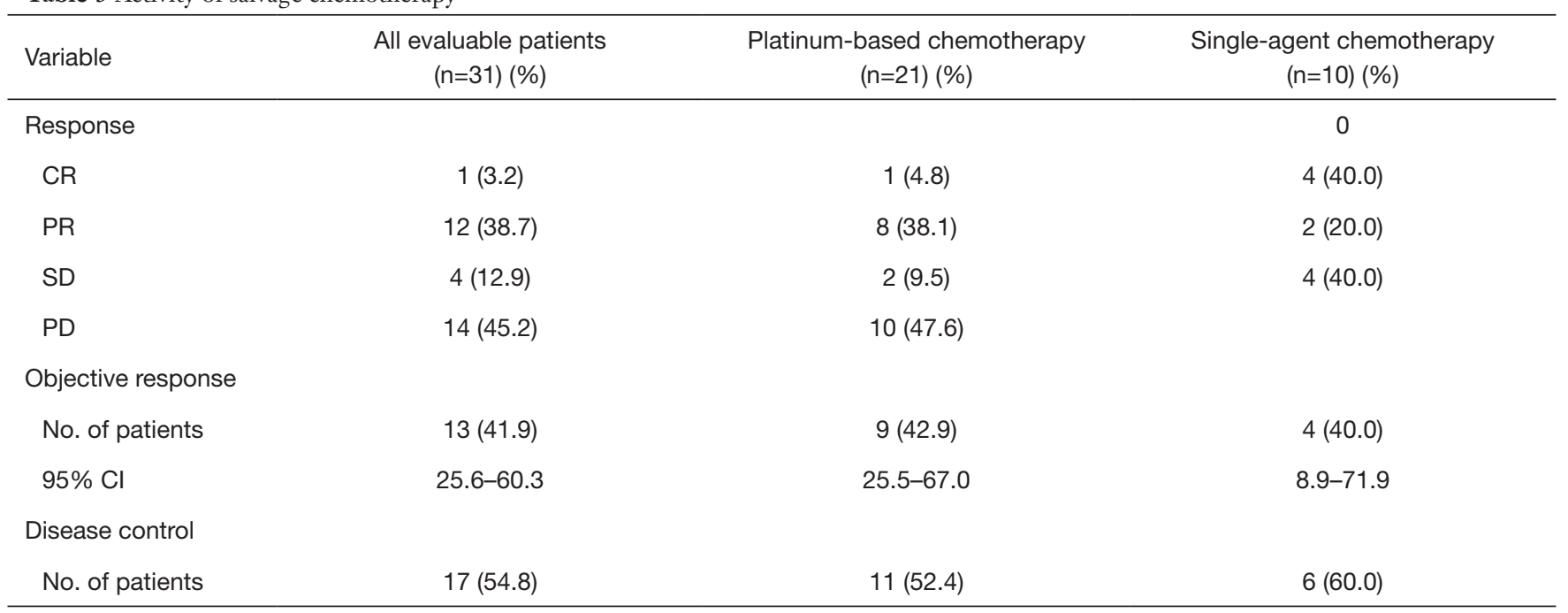

$\mathrm{CR}$, complete response; PD, progressive disease; PR, partial response; SD, stable disease; Cl, confidence interval.
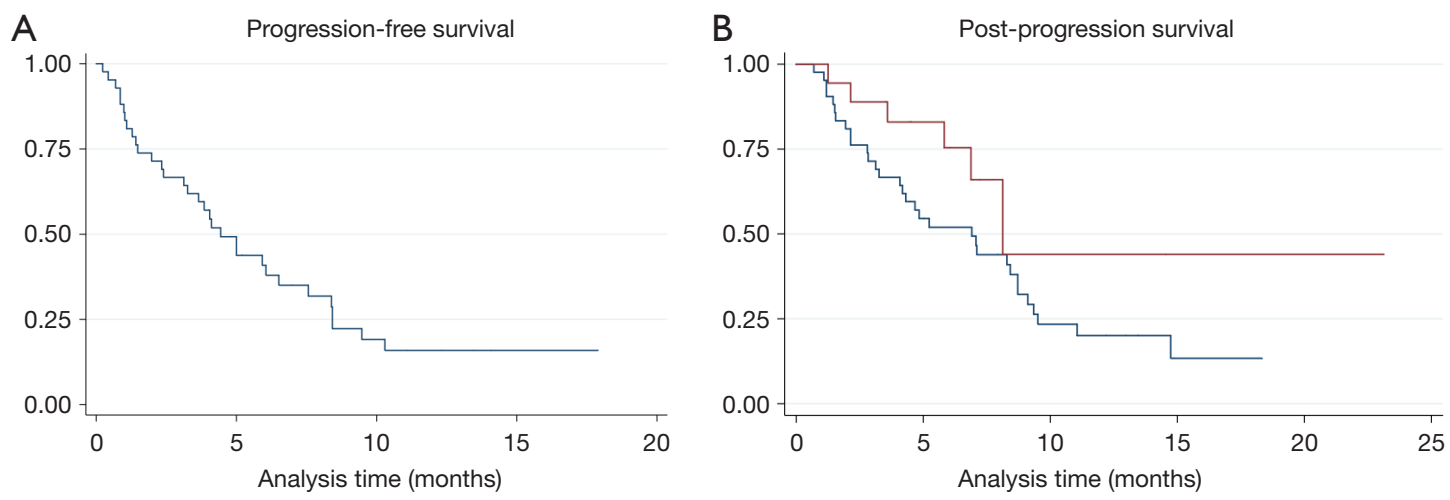

Salvage chemotherapy $\longrightarrow$ Pembrolizumab beyond progression

Figure 2 Survival curves. (A) Kaplan-Meier estimate of progression-free survival for patients treated with salvage chemotherapy; (B) Kaplan-Meier estimates of post-progression survival for patients treated with salvage chemotherapy (blue line) and for patients treated with pembrolizumab beyond progression (red line).

care for this group of patients, either pembrolizumab as single-agent or pembrolizumab in combination with chemotherapy (6-8).

An interesting issue is whether prior exposure to PD-1 axis inhibitor therapy may affect response to salvage chemotherapy (13). Some authors have reported a high anticancer activity for chemotherapy administered immediately after anti-PD-1/PD-L1 agents across multiple cancers (14-17). With regard to NSCLC, Park et al. found an outstanding response rate of $66.7 \%$ for platinum-based chemotherapy administered as salvage treatment after PD-1 axis inhibitors as opposed to $39.5 \%$ for platinum-based chemotherapy administered prior to anti-PD-1/PD-L1 agents (16). Of note, the same authors reported an outstanding ORR of $53 \%$ even for singleagent chemotherapy when administered after PD-1 axis inhibitors. Similarly, another study reported an ORR of $39 \%$ with single-agent docetaxel administered sequentially after anti-PD-1/PD-L1 agents (17). Likewise, our data showed an excellent activity for salvage chemotherapy, as we reported an ORR of $42.9 \%$ for platinum-based chemotherapy and an ORR of $40 \%$ for single-agent chemotherapy (Table 3). However, while prior studies mainly focused on heavily pretreated NSCLC patients 
Table 4 Patients who were treated with pembrolizumab beyond disease progression

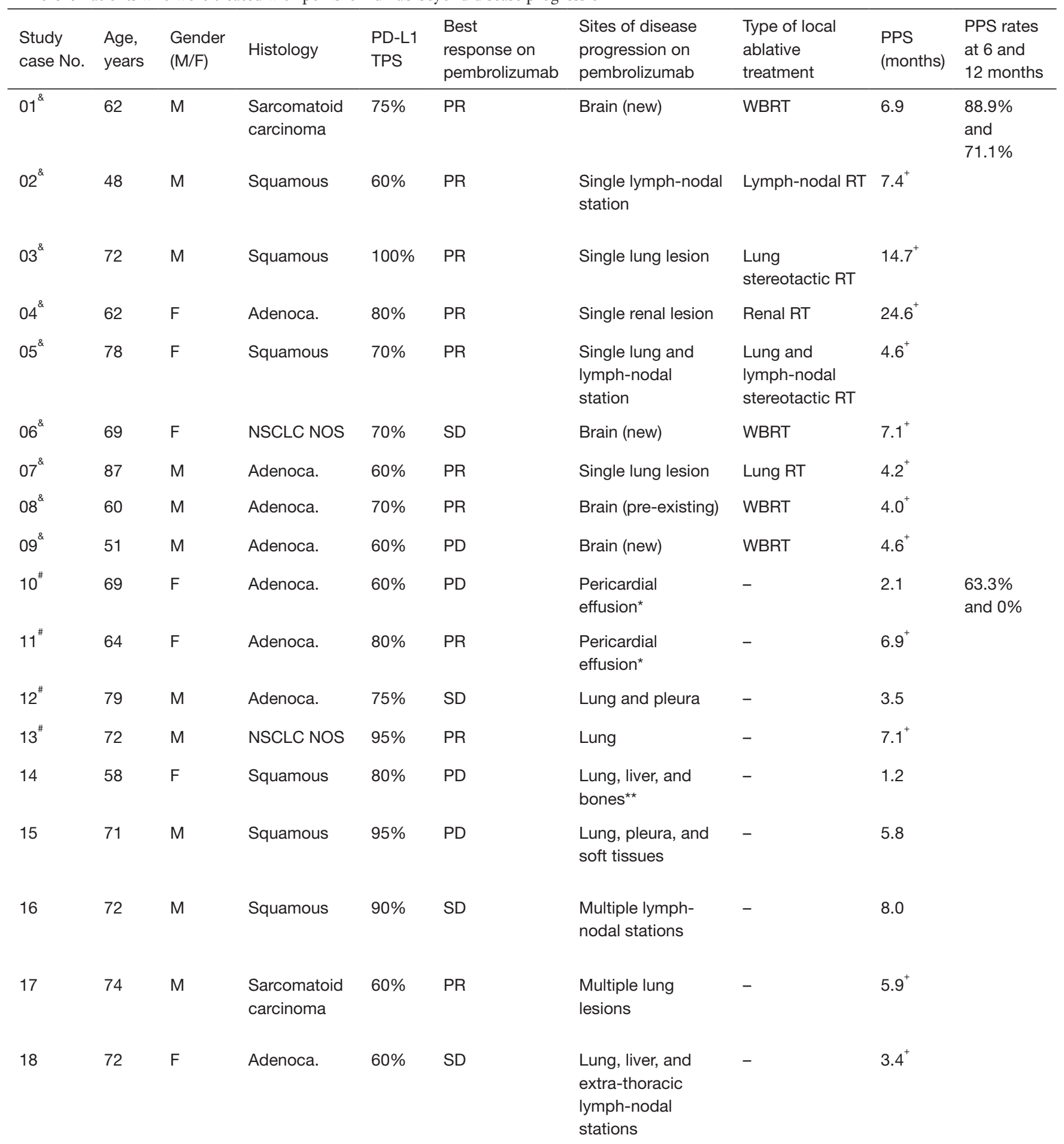

\footnotetext{
\& , patients with progressive disease at $\leq 2$ organ sites treated with local ablative radiotherapy; ", patients with progressive disease at $\leq 2$ organ sites; * , treated by pericardial drainage only; ${ }^{* *}$, palliative bone radiotherapy administered; ${ }^{+}$, sign indicates alive patients at the time of the last contact. Adenoca., adenocarcinoma; No., number; NSCLC, non-small cell lung cancer; NOS, not otherwise specified; PD, progressive disease; PR, partial response; PPS, post-progression survival; RT, radiotherapy; SD, stable disease; TPS, tumor proportion score; WBRT, whole brain radiotherapy.
} 
irrespective of $\mathrm{PD}-\mathrm{L} 1$ expression, the present analysis suggests that salvage chemotherapy could also be highly effective when administered after first-line pembrolizumab in PD-L1 $\geq 50 \%$ advanced NSCLC patients, with an ORR of $41.9 \%$, a disease control rate of $54.8 \%$ and a median PFS of 4.5 months (Table 3, Figure $2 A$ ).

Of note, responses have been reported by some authors in patients who were re-challenged with PD-1 axis inhibitors after a treatment break $(9,18)$. In addition, two retrospective series showed survival benefit in patients who were treated with PD-1 axis inhibitors beyond progression compared to those whose treatment was stopped at the time of progression or those who switched to other anticancer treatments $(10,11)$. In this study we found that pembrolizumab beyond progression could be beneficial in select patients, as no significant differences were noted in terms of PPS between patients treated with salvage chemotherapy and those who received pembrolizumab beyond progression (6.9 versus 8.1 months, respectively, $\mathrm{P}=0.08$ ) (Figure $2 B$ ). As expected, the majority of patients who were treated with pembrolizumab beyond progression had experienced either PR or SD (14 out of 18 patients, $77.8 \%$ ) as best response to pembrolizumab and had undergone PD in $\leq 2$ organ sites ( 13 out of 18 patients, $72.2 \%$ ) (Table 4). Importantly, in 9 of the 13 patients $(69.2 \%)$ with oligoprogressive disease in $\leq 2$ organ sites the benefits from pembrolizumab beyond progression were maximized through the addition of local ablative radiotherapy, with a reported 6- and 12-month PPS of $88.9 \%$ and $71.1 \%$, respectively. Consistently, a small retrospective study involving 26 patients with acquired resistance to anti-PD-1/PD-L1 agents found an outstanding 2 -year survival rate of $92 \%$ for the 15 patients who were managed with the addition of local ablative therapies (mainly radiotherapy) for oligoprogressive disease with or without treatment with PD-1 axis inhibition beyond progression (9). Whether local ablative radiotherapy may have contributed to the control of occult disease through additional abscopal effects in the nine patients from our series who achieved a long-term survival beyond progression is a fascinating hypothesis, and ongoing clinical studies are currently evaluating the abscopal potential of local tumor radiation with PD-1 axis inhibitor therapy (19).

A few limitations should be acknowledged in the present study. First of all, this is a retrospective study with potential biases affecting the results. Second, the number of patients included is relatively small. Third, patients treated with salvage chemotherapy and pembrolizumab beyond progression are not formally comparable because of lack of randomization, as those who continued pembrolizumab beyond progression could have had a better clinical status than patients who were switched to salvage chemotherapy. However, the fact that patients in the salvage chemotherapy group were clinically fit in order to receive salvage chemotherapy suggests that they were not expected to undergo rapid clinical deterioration at the time of treatment initiation. Lastly, it may be argued that the benefits of pembrolizumab beyond progression could be ascribed to the existence of an atypical response pattern termed pseudoprogression, which consists of an initial radiological assessment meeting conventional response criterion for PD, followed by a decrease in tumor burden at subsequent radiological assessments (20). Nevertheless, while pseudoprogression seems to be relatively common in melanoma, it is much less frequent in NSCLC, occurring in approximately $2 \%$ of patients (21). In addition, since most of patients treated with pembrolizumab beyond progression had experienced a PR or SD as best response to prior pembrolizumab, it seems likely that our patients were experiencing acquired resistance rather than pseudoprogression. Despite these limitations, this study has the strength of describing in a real-life scenario how patients with $\mathrm{PD}-\mathrm{L} 1 \geq 50 \%$ advanced NSCLC perform on subsequent anticancer therapies at the time of failure on first-line pembrolizumab. This remains a relevant question as not all of these patients will be treated upfront with the combination of PD-1 axis inhibitor therapy and chemotherapy.

In conclusion, we showed that chemotherapy is a highly effective rescue treatment for PD-L $1 \geq 50 \%$ advanced NSCLC patients who failed first-line pembrolizumab. On the other hand, continuation of pembrolizumab beyond progression should be considered in select cases depending on the benefit on prior pembrolizumab and/or number of progressive organ sites and lesions, along with local ablative radiotherapy which may add to the overall clinical benefit of such a strategy in oligoprogressive cases.

\section{Acknowledgments}

None.

\section{Footnote}

Conflict of Interest: This work was presented in abstract form at World Conference on Lung Cancer 2019. Giulio Metro 
has had consulting/advisory relationship with BoehringherIngelheim; Giuseppe Banna has had consulting/advisory relationships with Boehringher-Ingelheim, Janssen-Cilag, Roche; Athina Christopoulou has had consulting/advisory relationships with Bristol Myers Squibb, Merck, Novartis, Pfizer, Roche, Sanofi; Antonio Calles received honorary/ consulting fees from AstraZeneca, Boehringher-Ingelheim, Bristol Myers Squibb, Eli Lilly, Merck, Novartis, Pfizer, Roche; Helena Linardou has had consulting/advisory relationships with AstraZeneca, Boehringher-Ingelheim, Bristol Myers Squibb, Merck, Novartis, Roche; Paris Kosmidis has had consulting/advisory relationships with Bristol Myers Squibb, Merck, Novartis, Pfizer. The other authors have no conflicts of interest to declare.

Ethical Statement: The authors are accountable for all aspects of the work in ensuring that questions related to the accuracy or integrity of any part of the work are appropriately investigated and resolved. Patients provided written informed consent for the collection of clinical outcomes information. Patients provided written informed consent for the collection of clinical outcomes information. All procedures were performed in accordance with the ethical standards of the institutional and/or national research committee and with the 1964 Helsinki Declaration and its later amendments.

\section{References}

1. Toschi L, Rossi S, Finocchiaro G, et al. Non-small cell lung cancer treatment (r)evolution: ten years of advances and more to come. Ecancermedicalscience 2017;11:787.

2. Borghaei H, Paz-Ares L, Horn L, et al. Nivolumab versus docetaxel in advanced non-squamous non-small cell lung cancer. N Engl J Med 2015;373:1627-39.

3. Brahmer J, Reckamp KL, Baas P, et al. Nivolumab versus docetaxel in advanced squamous-cell non-small-cell lung cancer. N Engl J Med 2015;373:123-35.

4. Herbst RS, Baas P, Kim DW, et al. Pembrolizumab versus docetaxel for previously treated, $\mathrm{PD}-\mathrm{L} 1$-positive, advanced non-small-cell lung cancer (KEYNOTE-010): a randomised controlled trial. Lancet 2016;387:1540-50.

5. Rittmeyer A, Barlesi F, Waterkamp D, et al. Atezolizumab versus docetaxel in patients with previously treated non-small-cell lung cancer (OAK): a phase 3, openlabel, multicentre randomised controlled trial. Lancet 2017;389:255-65.

6. Reck M, Rodríguez-Abreu D, Robinson AG, et al.
Pembrolizumab versus chemotherapy for PD-L1positive non-small-cell lung cancer. N Engl J Med 2016;375:1823-33.

7. Reck M, Rodríguez-Abreu D, Robinson AG, et al. Updated analysis of KEYNOTE-024: pembrolizumab versus platinum-based chemotherapy for advanced nonsmall-cell lung cancer with PD-L1 tumor proportion score of 50\% or greater. J Clin Oncol 2019;37:537-46.

8. Addeo A, Banna GL, Metro G, Di Maio M. Chemotherapy in combination with immune checkpoint inhibitors for the first-line treatment of patients with advanced non-small cell lung cancer: a systematic review and literature-based meta-analysis. Front Oncol 2019;9:264.

9. Gettinger SN, Wurtz A, Goldberg SB, et al. Clinical features and management of acquired resistance to PD-1 axis inhibitors in 26 patients with advanced non-small cell lung cancer. J Thorac Oncol 2018;13:831-9.

10. Gandara DR, von Pawel J, Mazieres J, et al. Atezolizumab treatment beyond progression in advanced NSCLC: results from the randomized, phase III OAK Study. J Thorac Oncol 2018;13:1906-18.

11. Ricciuti B, Genova C, Bassanelli M, et al. Safety and efficacy of nivolumab in patients with advanced non-smallcell lung cancer treated beyond progression. Clin Lung Cancer 2019;20:178-185.e2.

12. McDonald F, Hanna GG. Oligoprogressive Oncogeneaddicted Lung Tumours: Does Stereotactic Body Radiotherapy Have a Role? Introducing the HALT Trial. Clin Oncol (R Coll Radiol) 2018;30:1-4.

13. Saleh K, Khalifeh-Saleh N, Kourie HR, et al. Do immune checkpoint inhibitors increase sensitivity to salvage chemotherapy? Immunotherapy 2018;10:163-5.

14. Dwary AD, Master S, Patel A, et al. Excellent response to chemotherapy post immunotherapy. Oncotarget 2017;8:91795-802.

15. Szabados B, van Dijk N, Tang YZ, et al. Response rate to chemotherapy after immune checkpoint inhibition in metastatic urothelial cancer. Eur Urol 2018;73:149-52.

16. Park SE, Lee SH, Ahn JS, et al. Increased response rates to salvage chemotherapy administered after PD-1/PDL1 inhibitors in patients with non-small cell lung cancer. J Thorac Oncol 2018;13:106-11.

17. Schvartsman G, Peng SA, Bis G, et al. Response rates to single-agent chemotherapy after exposure to immune checkpoint inhibitors in advanced non-small cell lung cancer. Lung Cancer 2017;112:90-5.

18. Garon EB, Hellmann MD, Rizvi NA, et al. Five-year overall survival for patients with advanced non-small- 
cell lung cancer treated with pembrolizumab: results from the phase I KEYNOTE-001 study. J Clin Oncol 2019;37:2518-27.

19. Walker J, Loo BW Jr. radiotherapy and immunotherapyshining further together. JAMA Oncol 2019. [Epub ahead of print].

20. Hodi FS, Hwu WJ, Kefford R, et al. Evaluation of immune-related response criteria and RECIST v1.1 in patients with advanced melanoma treated with pembrolizumab. J Clin Oncol 2016;34:1510-7.

21. Katz SI, Hammer M, Bagley SJ, et al. Radiologic pseudoprogression during anti-PD-1 therapy for advanced non-small cell lung cancer. J Thorac Oncol 2018;13:978-86.
Cite this article as: Metro G, Addeo A, Signorelli D, Gili A, Economopoulou P, Roila F, Banna G, De Toma A, Rey Cobo J, Camerini A, Christopoulou A, Lo Russo G, Banini M, Galetta D, Jimenez B, Collazo-Lorduy A, Calles A, Baxevanos P, Linardou H, Kosmidis P, Garassino MC, Mountzios G. Outcomes from salvage chemotherapy or pembrolizumab beyond progression with or without local ablative therapies for advanced non-small cell lung cancers with $\mathrm{PD}-\mathrm{L} 1 \geq 50 \%$ who progress on first-line immunotherapy: real-world data from a European cohort. J Thorac Dis 2019;11(12):4972-4981. doi: 10.21037/jtd.2019.12.23 